Rev. Elev. Méd. Vét. Pays trop., 1966, 19, 4 (511-26).

\title{
Etude du pouvoir anthelminthique du Tetramisole (16.535 R. P.) sur divers helminthes du zébu de la République du Tchad *
}

\author{
par M. GRABER \\ Institut d'Elevage ef de Médecine Vétérinaire des Pays Tropicaux \\ Laboratoire Farcha-Fort-Lamy - République du Tchad \\ Rhône-Poulenc, 22 avenue Montaigne, Paris
}

\begin{abstract}
RÉsumÉ
L'auteur, après avoir effectué des essais thérapeutiques sur 107 zébus, jeunes et adultes, originaires des régions Ouest de la République du Tchad estime que la dose de $5 \mathrm{mg} / \mathrm{kg}$ de Tetramisole administrée sans dièfe par la voie buccale ou par la voie sous-cutanée suffit d̀ détruire dans la proportion de $90-95$ p. 100 les associations à base de Bosicala radiatum, Bunostomum phlebotomum, Hoemoncus, contortus, Cooperia pectinata et Cooperia punctata adultes môrs ou immatures.

Les formes $L_{4}$ de Bosicola radiatum enkystées dans la muqueuse intestinale résistent à tout traitement. Quelques formes larvaires de Cooperia persistent également.

Le médicament est relativement peu toxique pour le zébu, puisque le coefficient chimiothérapique va de 8-10 (voie sous-cutanée) à 15 (voie buccale). A la dose thérapeutique, bien supporlée, l'amélioration de l'état général est très rapide et, au bout de sept semaines, dans de très mauvaises conditions alimentaires, le gain de poids est de $+17,6$ p. 100 (témoins $:+12,9$ p. 100). Le 16.535 R. P. présente donc un intérêt économique indéniable.
\end{abstract}

\section{INTRODUCTION}

Depuis longtemps déjà, Chimistes ef Biologistes, dans la lutte contre les Nématodes du gros bétail, ont cherché à mettre ou point un médicament parfait. Un grand nombre d'antheiminthiques ont été successivement essayés avec des fortunes diverses.

Dans cet ordre d'idées, le Laboratoire de Farcha a expérimenté, au cours de ces dix dernières années, plusieurs dizaines de corps,

(*) Remis pour publication le 28-4-1966. en ne perdant pas de vue que, dans des pays tropicaux secs à élevage nomade ou seminomade comme le Tchad, un bon traitement doit obéir aux impératifs suivants :

- L'existence au départ d'un troupeau fortément anémié le rend plus sensible au médicament qui devra alors être dépourvu de toute toxicité.

- La présence de Nématodes associés milite en faveur de l'emploi d'un anthelminthıque polyvalent à très large spectre d'activité, tant sur les formes adultes que sur les formes immatures. 
- La diète préalable est à proscrire : les troupeaux seront traités immédiatement là où ils se trouvent.

- L'administration du médicament devra être effectuée par un personnel non spécialisé, le plus souvent par le propriétaire du troupeau lui-même.

- Le coôt de l'opération devra être faible et l'accroissement du rendement du troupeau après traitement démontré.

Le dernier anthelminthique en date, le Thiabendazole, s'il n'obéit pas entièrement à toutes ces règles, constitue un progrès très net par rapport à ce qu'il était possible de recommander auparavant (GRABER, 1965).

Tout récemment, THIENPONT et Coll. (1966) ont signalé que le di (tetrahydro-2, 3, 5, 6, Phenyl-6 imidazo (2-1) b thiazole, Chlorhydrate ou Tétramısole (nom générıque) ou 16.535 R. P. pouvait être considéré comme un anthelminthique de très grande valeur, ce qui, chez le mouton, a déjà été confirmé par WALLEY (1966) et GRABER (1966).

Le but du présent travail (*) est de donner un aperçu général de la valeur antiparasitaıre et économique du médicament à l'égard des Nématodes bovins les plus fréquents en Afrıque centrale.

\section{MATÉRIEL ET MÉTHODE}

\section{A. - L'Anthelminthique}

Le Tétramisole, $\mathrm{C}_{11} \mathrm{H}_{12} \mathrm{~N}_{2} \mathrm{SHCL}$, a comme formule :

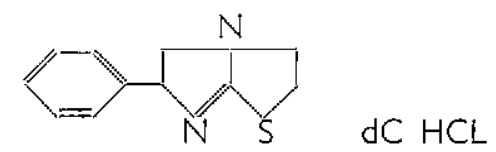

Il se présente sous l'aspect d'une poudre sans odeur, blanche, amorphe et de saveur aigredouce. Le poids moléculaire est de 240,753 et le point de fusion est atteint à $260^{\circ} \mathrm{C}$. La poudre - jusqu'à plus ample informé - paraît stable dans des conditions tropicales sévères $\left(+25{ }^{\circ} \mathrm{C}\right.$ à $+37^{\circ} \mathrm{C}$ pendant plus d'un an). Le Chlorhydrate titre 84,85 p. 100 de produit actif de base.

(*) Réalisé ò la demande de la société RhônePoulenc.
Il est soluble dans l'eau ( $1 \mathrm{~g}$ dans $4 \mathrm{ml})$ et l'éthanol $(1 \mathrm{~g}$ dans $70 \mathrm{ml})$. Mis au point par Janssen Pharmaceutica (*), le médicament est encore connu sous les noms de Ripercol (Belgique) ef de Nilwerm (**), et de Memicine (***).

II va être incessamment commercialisé en France.

\section{B. - LES ANIMAUX D'EXPÉRIENCE}

107 animaux originaires du Chari-Baguirmi et du Kanem (Ovest-Tchad) et pesant de 50 à 300 kilogrammes ont été utilisés de la façon suivante:

Essais thérapeutiques proprement dits : 57. Essais de toxicité : 20.

Témoins : 20.

Essais sur le terrain : 10.

Ils comprenaient :

28 vaches âgées.

79 bouvillons de 5 à 18 mois.

La plupart d'entre eux' hébergeaient à l'état naturel un grand nombre d'Helminthes (95 p. 100) (voir tableau).

Ces Helminthes étaient associés, dans 85 p. 100 des cas, par 2, 3 ou même 5 espèces différentes. Aussi, l'état des animaux était-il des plus médiocres chez les bouvillons les plus jeunes et chez les vaches les plus âgées.

Les essals ont été menés en six étapes de janvier 1965 d̀ avril 1966 (janvier, mai, juin, novembre-décembre 1965 ; mars-avril 1966), ce qui a permis de suivre le comportement des animaux aux époques favorables (d'août à février) et aux époques défavorables (de mars à juiilet).

La saison des pluies 1965 ayant été, au Tchad, particulièrement déficitaire, la repousse des pâturages a été faible, d'où un certain manque de fourrage à partir de février 1966 avec comme conséquence amaigrıssement général des effectifs. Les essais de toxicité effectués en avril 1966 sur des bouvillons profondément amaigris donnent des indicatıons précises sur la résistance des animaux au Tétramisole administré à fortes doses.

(*) Beerse-Belgıque.

(**) Imperial Chemical Industries limited.

(***) Spécia et Roger Bellon. 


\begin{tabular}{|c|c|c|c|c|c|c|}
\hline Espèces parasites & $\frac{\text { Animaux traités }}{(64)}$ & $\begin{array}{l}\text { Animaux tém } \\
\text { (20) }\end{array}$ & oins & \multicolumn{3}{|c|}{ (84 motal } \\
\hline 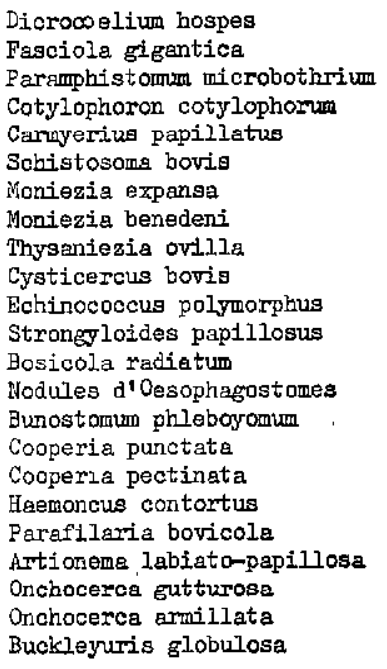 & $\begin{array}{r}- \\
19 \\
14 \\
- \\
3 \\
40 \\
- \\
1 \\
2 \\
8 \\
1 \\
6 \\
35 \\
6 \\
16 \\
35 \\
15 \\
- \\
25 \\
29 \\
27 \\
5\end{array}$ & 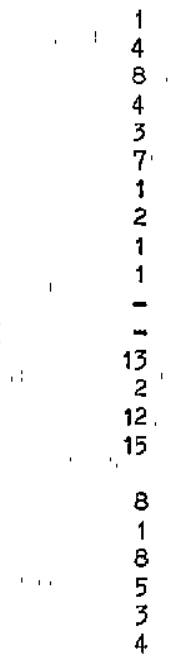 & T & & $\begin{array}{r}1 \\
23 \\
22 \\
4 \\
6 \\
1 \quad 47 \\
1 \\
3 \\
3 \\
9 \\
1 \\
6 \\
48 \\
8 \\
28 \\
50 \\
\\
23 \\
1 \\
33 \\
34 \\
30 \\
9\end{array}$ & \\
\hline
\end{tabular}

\section{C. - MÉTHODE}

Elle est rigoureusement calquée sur celle qui a été décrite dans un précédent articlè (GRABER, 1966). Elle met l'accent sur l'importance qu'il $y$ a, dans ce genre d'essais, d̀ travailler sur autopsies avec un grand nombre de témoins, les examens coproscopiques et les cultures d'œufs ne servant qu'à orienter l'expérience.

\section{Avant traitement.}

Chaque animal est mis en observation durant 48 heures ef divers examens (coproscopiques et hématologıques) sont pratıqués dans le but de situer l'importance exacte du parasitisme. Parallèlement, des cultures d'œufs permettent de savoir à quels Nématodes on a affaire.

'En fonction des renseignements aınsi obtenus', les lots sont constitués : ils comprennent un tiers d'animaux très parasités, un tiers d'animaux moyennement parasités et un tiers d'anımaux faiblement parasités.

\section{Traitement.}

Lo totalité des essais a été effectuée sans préparation, c'est-à-dire sans diète préalable. Les animaux ont été marqués et placés dans des stalles individuelles cimentées.
L'anthelminthique a été administré de deux manières :

- par la voie buccale « à la bouteille»,

- par la vole sous-cutanée. Le 16.535 R. P. est alors dissous dans de l'eau distillée stérile. II existe divers types de solution ( 5 p. $100 ; 9$ p. 100 ; 10 p. 100). 'C'est cette dernière qui a été employée. A $30 \circ \mathrm{C}$, il faut environ une demi-heure pour que les derniers cristaux disparaissent. La solution, sans dépôt, est alors injectée derrière l'épaule avec les précautions habituelles (asepsie) : dans ces conditions, 'il ne se produit, au point d'inoculatıon ni ınflammatıon, ni nécrose au bout de 15-18 jours, alors que certains auteurs en font état (WALLEY, 1966).

Il est bien entendu que les doses administrées sont calculées en produif de base pur, ce qui nécessite une correction, puisque l'anthelmınthique est sous forme de Chlorhydrate $(1 \mathrm{~g}$ de produit pur représente $1,1765 \mathrm{~g}$ de Chlorhydrate).

\section{Après troitement.}

Sur chaque animal, il a été procédé pendant 8 à 10 jours :

a) au ramassage des crottes trois fois par jour. Elles ont été broyées dans un mince filet d'eau et soigneusement examınées, de manière ò 
faire apparaître les Helminthes - Cestodes et Nématodes - évacués. Ceux-ci ont été comptés, pesés et déterminés,

b) à des examens coproscopiques journaliers par la méthode de sédimentation de Brumpt, la plus simple à mettre en ceuvre dans ce pays. La comparaison entre la moyenne du nombre d'œufs au gramme avant traitement, après traitement et le jour de l'autopsie permet d'avoir un premier aperçu de l'efficacité du médicament,

c) à des cultures d'œufs en boîtes de Pétri sur papier buvard. Arbitrairement, on évalue chaque jour le nombre de larves $L_{3}$ rencontrées dans une goutte de suspension aqueuse provenant des boîtes de Pétri. Pour chaque lot, la comparaison entre la moyenne du nombre de larves avant et après le traitement complète en général les éléments 'd'appréciation fournis par l'examen coproscopique. Pour certains Nématodes digérés dans l'intestin à la suite du traitement au Tétramisole, la méthode est absolument indispensable (Strangyloides ef Cooperia).

\section{Autopsie.}

Passé le délai de 10 jours, les animaux sont sacrifiés. Les Helminthes demeurés en place sont récoltés ce qui ne pose pas de problème pour les grands Cestodes, Bosicola radiatum. Bunostomum phlebotomum. Haemoncus contorius et Buckleyuris globulosa.

Lorsqu'il s'agit de petits Nématodes (Strongyloïdes et Cooperia), it importe de gratter la muqueuse duodénale sur une longueur de 30 à $50 \mathrm{~cm}$. L'examen au microscope du produit de raclage placé entre lame et lamelle confirme ou non la présence des Nématodes en cause.

Pour les formes larvaires $\mathrm{L}_{4}$ intranodulaıres de' Bosicola radiatum, chaque nodule cæcal est ouvert et le contenu, écrasé entre lame et lamelle, est regardé au microscope.

\section{RÉSULTATS \\ A. - LES TÉMOINS (Tableau I) \\ B. - ACTION SUR LES TRÉMATODES}

Aux doses employées, que ce soit par la voie buccale ou par la voiesous-cutanée, le 16.535 R.P. est dépourvu de toute activité à l'égard de
Fasciolo gigantica des canaux biliaires, de Paramphistomum microbothrium et de Cormyerius popillatus de la panse, de Schistosoma bovis des veines hépatiques et mésentériques (Tableau II).

\section{C. - ACTION SUR LES CESTODES}

(Tableau III)

Là encore, le Tétramisole est, à $5 \mathrm{mg} / \mathrm{kg}$. totalement inactif sur les Anoplocephalidae de l'intestin (Moniezia benedeni et Thysoniezia ovilla) ef sur Cysticercus bovis qui, après traitement, s'évagine parfaitement dans la bile portée à la température de l'éṫuve $\left(+39^{\circ} \mathrm{C}\right)$.

\section{D. - ACTION SUR LES NÉMATODÉS}

10 Sur les formes adultes mûres ou immatures. Tableau No IV : examens coproscopiques.

Tableau No $V$ : Strongyloïdes papillosus.

Tableau No VI : Bunostomum phlebotomum.

Tableau No VII : Bosicolo radiatum adultes mûrs.

Tableau No VIII: Bosicola radiatum adultes immatures.

Tableau' No IX : Cooperia punctato et Cooperia pectinata.

Tableau No $X$ : Haemoncus contorfus.

Tableau No $X I$ : Filariidae.

$2^{\circ}$ Sur les formes intranodulaires $L_{4}$ de Bosicola radiatum (Tableau No XII).

$3^{\circ}$ Sur les formes larvaires immatures de Cooperio punctata et de Cooperia pectinata.

La dose de $5 \mathrm{mg} / \mathrm{kg}$ par la voie buccale laisse subsister un petit nombre de formes larvaires de Cooperia punctota et de Cooperia pectinota (5 à 10 par animal). II ne reste plus aucun parasite lorsque le médicament est administré par la voie sous-cutanée: Dans les deux cas, les animaux avaient été placés sur des parcours riches en larves de Cooperia et traités 25 jours plus tard.

$4^{0}$ Discussion.

a) Le Tétramisole est dépourvu de toute activité à l'égard d'Artionema labiato-papillosa du péritoine, d'anchocerca gutturosa du ligament cervical ef d'Onchocerca armillata de l'aorte. 
TABLEAO HOI

Les témoins

\begin{tabular}{|c|c|c|c|c|c|}
\hline Espèces en causes & $\underset{(3+)}{J_{a n v i e r}} 65$ & $\begin{array}{c}\text { Maj } 65 \\
\text { (2) }\end{array}$ & $\mathrm{J}_{(4)} 65$ & $\begin{array}{l}\text { Novembre } \\
\text { Décembre } \\
1965 \text { (5) }\end{array}$ & $\begin{array}{c}\text { Mers } \\
\text { Avril } \\
1966(6)\end{array}$ \\
\hline $\begin{array}{l}\text { Dicrocoelium hospes } \\
\text { Fasciola gigantica } \\
\text { P. microbothrium } \\
\text { Cotylophoron cotylophorum } \\
\text { Carmyerius papillatus } \\
\text { Schistosoma bovis } \\
\text { Moniezia expansa } \\
\text { Moniezia benedeni } \\
\text { Thysaniezia ovilia } \\
\text { Gysticercus bovis } \\
\text { Bosicola radiatuma } \\
\text { adultes mûrs } \\
\text { Bosicola radiatum adultes } \\
\text { immatures } \\
\text { Oesophagostomes larvaires } \\
\text { Bumostomum phlebotomum } \\
\text { Cooperia punctata } \\
\text { Cooperia pectinata } \\
\text { Haemoncus contortus } \\
\text { Parafilaria bovicola } \\
\text { Artionema labiato-papillosa } \\
\text { Onchocerca gqtturosa } \\
\text { Onchocerca armillata } \\
\text { Buckleyuris globulasa }\end{array}$ & $\begin{array}{l}\overline{-} \\
\overline{-} \\
\overline{-} \\
\overline{1}++ \\
0.5 \\
- \\
- \\
7^{+1+} \\
\overline{1} \\
1 \\
39 \\
166 \\
40 \\
\overline{1} \\
- \\
+ \\
1\end{array}$ & 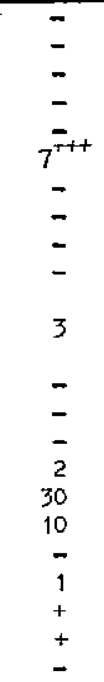 & $\begin{array}{c}- \\
23 \\
1,1+ \\
- \\
31 \\
- \\
= \\
5 \\
19 \\
- \\
- \\
3 \\
- \\
3 \\
104 \\
- \\
2 \\
+ \\
-\end{array}$ & $\begin{array}{c}\overline{8} \\
14 \\
- \\
1 \\
7 \\
- \\
- \\
160 \\
- \\
11 \\
3 \\
2 \\
34 \\
9 \\
19 \\
24 \\
- \\
2 \\
+ \\
- \\
-\end{array}$ & $\begin{array}{c}2 \\
9 \\
1,6 \\
1,5 \\
1 \\
12 \\
- \\
43 \\
- \\
- \\
7 \\
7 \\
25 \\
- \\
30 \\
118 \\
50 \\
15 \\
2 \\
22 \\
- \\
+ \\
3\end{array}$ \\
\hline
\end{tabular}

+ Nombre d'animaur utilisés

t+r Cestodes et Paramphistomes : poits moyen de parasites (en g.)

H+ Némitodes, Schistosomes, Fasciola et Dicrocoelium : moyenne du nombre de parasites.

TABLEAU NOII

Trématodes - Nombre d'arimaux déparagités après traitement au Tétramisole

\begin{tabular}{|c|c|c|c|c|}
\hline $\begin{array}{l}\text { Doges } \\
\mathrm{mg} / \mathrm{kg}\end{array}$ & F. gigantica & P. microbothrium & C. papillatus & S. bovls \\
\hline $\begin{array}{c}\text { Voie buccele } \\
5 \\
10 \\
20 \\
40\end{array}$ & $\begin{array}{c}0 \text { sur } 4 \\
0 \text { sur } 1 \\
-\end{array}$ & $\begin{array}{l}0 \text { sur } 9 \\
- \\
0 \text { sur } 1 \\
0 \text { sur } 1\end{array}$ & $\begin{array}{c}0 \text { sur } 2 \\
0 \text { gur } 1 \\
- \\
-\end{array}$ & $\begin{array}{lll}0 & \text { sur } & 16 \\
0 & \text { sur } & 2 \\
0 & \text { sur } & 3 \\
0 & \text { sur } & 1\end{array}$ \\
\hline $\begin{array}{c}\text { Vole souf-cut } \\
5 \\
8 \\
10\end{array}$ & 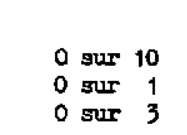 & $\begin{array}{rll}0 & \text { sur } & 1 \\
0 & \text { sur } & 1 \\
0 & \text { sur } & 1\end{array}$ & $\overline{-}$ & 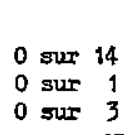 \\
\hline
\end{tabular}

TABLBAO HOIII

Cestodes - Nombre d'animaux déparasités après traitement au Tétramisole.

\begin{tabular}{|c|c|c|c|c|}
\hline $\begin{array}{c}\text { Doses } \\
\text { mg/kg }\end{array}$ & M. benedeni & T. ovilla & C. bovis & E. polymorphus \\
\hline $\begin{array}{c}\text { Vole buccale } \\
5\end{array}$ & - & & 0 sur 6 & sur 1 \\
\hline Voie sous-cutanée & 0 sur 1 & 0 sur 2 & 0 sur 2 & - \\
\hline 5
\end{tabular}


TABLEAD NOIV

Moyenue du nombre d'oeufs al gxamme de metière fécale.

\begin{tabular}{|c|c|c|c|c|c|c|}
\hline \multirow{2}{*}{$\begin{array}{l}\text { Dases } \\
\mathrm{mg} / \mathrm{kg}_{\mathrm{g}}\end{array}$} & \multicolumn{2}{|c|}{ svant traitement } & \multicolumn{2}{|c|}{ Après traitement } & \multicolumn{2}{|c|}{ dernter jour } \\
\hline & Strongles** & Stronger lotdes & Strongles & Strongyloides & Strongles & Strongrloiden \\
\hline $\begin{array}{l}\text { Vole buccalo } \\
5 \\
10 \\
20\end{array}$ & $\begin{array}{l}41 \\
26 \\
28\end{array}$ & $\begin{array}{l}- \\
-\end{array}$ & $\begin{array}{l}0 \\
0 \\
0\end{array}$ & $\begin{array}{l}- \\
-\end{array}$ & $\begin{array}{l}0 \\
0 \\
0\end{array}$ & $\begin{array}{l}- \\
-\end{array}$ \\
\hline $\begin{array}{l}\text { Voie sous-cut } \\
5 \\
\text { B }\end{array}$ & $\begin{array}{r}38 \\
315\end{array}$ & $=$ & $\begin{array}{r}1 \\
52\end{array}$ & $\overline{-}$ & 0 & $=$ \\
\hline
\end{tabular}

* = 10 jours aprìa la fin du traitement.

** = Haemoncus, Eunostomes, Oesophagostcones, Cooperia.

TABLEAO NOV

Action du Tétramisole sur Strongrloides paplllosus adultes moro

\begin{tabular}{|c|c|c|c|c|c|}
\hline & & $\begin{array}{l}\text { Culture } \\
\text { Nombre total }\end{array}$ & $\begin{array}{l}\text { d'oeufs } \\
\text { de larves L3* }\end{array}$ & Autopsle & \\
\hline $\begin{array}{l}\text { Doses } \\
\mathrm{mg} / \mathrm{kg}\end{array}$ & $\begin{array}{c}\text { Nombre d'animaux } \\
\text { parasités }\end{array}$ & Avant traitement & Après traitenext & $\begin{array}{l}\text { Nombre d'arimalux } \\
\text { encore paragitéa }\end{array}$ & $\begin{array}{l}\text { Epoque des } \\
\text { traitements }\end{array}$ \\
\hline $\begin{array}{c}\text { Voie } \\
5\end{array}$ & e. & 43 & 0 & 0 & $\begin{array}{l}\text { Novembre } \\
\text { Décembre } 1965\end{array}$ \\
\hline $\begin{array}{l}\text { Voie } \\
5\end{array}$ & utanée. & 10 & 0 & 0 & $\begin{array}{l}\text { Novembre } \\
\text { Décembre } 1965\end{array}$ \\
\hline
\end{tabular}

* = dans une goutte de suspension aqueuse provenant des boftes de Petri ayant servi aux coprocuitures.

TABLEAU NOVI

Action du Tétramisole sur Bunostonum phlebotomun adultes murs et 1mmatures

\begin{tabular}{|c|c|c|c|c|c|}
\hline $\begin{array}{l}\text { Doses } \\
\mathrm{mg} / \mathrm{kg}\end{array}$ & $\begin{array}{c}\text { Nombre d'enimaux } \\
\text { parasités }\end{array}$ & $\begin{array}{l}\text { Nombre de } \\
\text { Nématodes } \\
\text { expulgés }\end{array}$ & $\begin{array}{l}\text { Présence }(+) \text { ou } \\
\text { absence }(-) \text { de } \\
\text { parasites a } \\
\text { 1'autonsio }\end{array}$ & $\begin{array}{l}\text { Nombre d'animaux } \\
\text { totalement } \\
\text { déparasités } \\
\text { Efficacité }\end{array}$ & $\begin{array}{l}\text { Upoque des } \\
\text { traitements }\end{array}$ \\
\hline $\begin{array}{c}\text { Voie } \\
5 \\
10 \\
20 \\
40\end{array}$ & $\begin{array}{l}4 \\
3 \\
1 \\
1\end{array}$ & $\begin{array}{l}65 \\
11 \\
6 \\
2\end{array}$ & $\begin{array}{l}- \\
- \\
-\end{array}$ & $\begin{array}{l}4 \text { sur } 4 \\
3 \text { sur } 3 \\
1 \text { sur } 1 \\
1 \text { sur } 1\end{array}$ & $\begin{array}{l}\text { Décembre } 1965 \\
\text { Janvier et } \\
\text { Mai } 1965 \\
\text { Janvier } 1965 \\
\text { Janvier } 1965\end{array}$ \\
\hline \multicolumn{6}{|c|}{ Vole sous-cutsnée. } \\
\hline $\begin{array}{l}5 \\
10\end{array}$ & $\begin{array}{r}3 \\
1\end{array}$ & $\begin{array}{l}3 \\
1\end{array}$ & - & $\begin{array}{l}3 \text { sur } 3 \\
1 \text { sur } 1\end{array}$ & $\begin{array}{l}\text { Novembre } \\
\text { Décembre } 1965 \\
\text { Juin } 1965\end{array}$ \\
\hline
\end{tabular}


TABLEAD NOVII

Action du Tétremisole sur Bosicala radiatum aơultes nûrrs.

\begin{tabular}{|c|c|c|c|c|c|c|}
\hline $\begin{array}{l}\text { Doges } \\
\mathrm{mg} / \mathrm{kg}\end{array}$ & $\begin{array}{l}\text { Nombre } \\
\text { d'animaux } \\
\text { parasités }\end{array}$ & $\begin{array}{c}\text { Nombre } \\
\text { d'cesophagostomes } \\
\text { exp ulsés }\end{array}$ & $\begin{array}{l}\text { Présence (+) ou } \\
\text { absence (-) de } \\
\text { parasites à } \\
\text { l'autopsie }\end{array}$ & $\begin{array}{l}\text { Nombre d'animaux } \\
\text { totalement } \\
\text { déparasités }\end{array}$ & Efflcacité & $\begin{array}{c}\text { Epoque } \\
\text { des } \\
\text { traitements }\end{array}$ \\
\hline$\underset{5}{\text { Voine b }}$ & $\begin{array}{l}2 \\
1 \\
2\end{array}$ & $\begin{array}{l}220 \\
31 \\
29 \\
47\end{array}$ & $\begin{array}{l}- \\
m\end{array}$ & $\begin{array}{l}11 \text { sur } 11 \\
\end{array}$ & $160 \mathrm{p} .100$ & $\begin{array}{l}\text { Maj } 1965 \\
\text { Novembre } \\
\text { Décembre } 1965 \\
\text { Mara } 1966 \\
\text { Mai } 1965 \\
\text { Janvier } 1965 \\
\text { Janvier } 1965\end{array}$ \\
\hline \multicolumn{7}{|c|}{ Voie sous-cutanée. } \\
\hline $\begin{array}{r}5 \\
8 \\
10\end{array}$ & $\begin{array}{l}5 \\
1 \\
4\end{array}$ & $\begin{array}{r}49 \\
20 \\
6\end{array}$ & $\begin{array}{l}- \\
-\end{array}$ & $\begin{array}{l}5 \text { sur } 5 \\
1 \text { sur } 1 \\
4 \text { sur } 4\end{array}$ & $\begin{array}{l}100 \quad n \\
100 \quad n\end{array}$ & $\begin{array}{l}\text { Mat-Juin } 1965 \\
\text { Décembre } 1965 \\
\text { Juin } 1965 \\
\text { Juin 1965. }\end{array}$ \\
\hline
\end{tabular}

TABLEAU No VIII

sotion du Tétramisole sur les formes adultes immatures de Bosicala radiatum

\begin{tabular}{|c|c|c|c|c|c|}
\hline $\begin{array}{l}\text { Doses } \\
\mathrm{mg} / \mathrm{kg}\end{array}$ & $\begin{array}{c}\text { Nombre } \\
\text { d"animaux } \\
\text { paresités }\end{array}$ & $\begin{array}{c}\text { Nombre } \\
\text { d 'esophagostomes } \\
\text { expulsés }\end{array}$ & $\begin{array}{l}\text { Présence }(+) \text { ou } \\
\text { absence (-) de } \\
\text { parasites à } \\
\text { l'autopsie }\end{array}$ & $\begin{array}{l}\text { Nombre d'animaux } \\
\text { totalement } \\
\text { déparesités } \\
\text { Effilcacité }\end{array}$ & $\begin{array}{l}\text { Epoque } \\
\text { des } \\
\text { traitements }\end{array}$ \\
\hline \multicolumn{6}{|c|}{ Vole buccale. } \\
\hline 10 & 2 & 27 & - & $2 \sin 2$ & Janvier 1965 \\
\hline 40 & 1 & 1 & 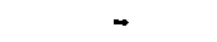 & 1 sur 1 & Janvier 1965 \\
\hline \multicolumn{6}{|c|}{ Vole squs-cutanée. } \\
\hline 5 & 3 & 14 & - & 3 sir 3 & Mat 1965 \\
\hline
\end{tabular}

TABTEAU NOIX

Action du Tétramisole sur Cooperia punctata et sur Cooperia pectinata adultes mors.



* = dang une goutte de auspension aqueuse provenant des bortes de Pétri ayant servi aur coprocultures. 
TABLEAU NOX

Action du Tétramisole sur Haemoncus contortus adultes môrs et immatures.

\begin{tabular}{|c|c|c|c|c|c|c|c|}
\hline \multirow{2}{*}{$\begin{array}{l}\text { Doses } \\
\mathrm{mg} / \mathrm{kg}\end{array}$} & \multicolumn{2}{|c|}{$\begin{array}{c}\text { Culture d'oeufs } \\
\text { Nombres de Larves } L_{3}^{*}\end{array}$} & \multirow{2}{*}{$\begin{array}{c}\text { Nombre } \\
\text { d'Haemoncus } \\
\text { expulsés }\end{array}$} & \multirow{2}{*}{$\begin{array}{c}\text { Présence (+) ou } \\
\text { absence ( }) \text { de } \\
\text { parasites a } \\
\text { I'autopsie }\end{array}$} & \multirow{2}{*}{$\begin{array}{l}\text { Nombre d'animaux } \\
\text { totalement } \\
\text { déparasités }\end{array}$} & \multirow[t]{2}{*}{ Efficacité } & \multirow{2}{*}{$\begin{array}{c}\text { Epoque } \\
\text { des } \\
\text { traitements }\end{array}$} \\
\hline & $\begin{array}{c}\text { Avant } \\
\text { traitement }\end{array}$ & $\begin{array}{c}\text { Apres } \\
\text { traitement }\end{array}$ & & & & & \\
\hline \multicolumn{2}{|c|}{ Voie buccale: } & - & & & & & \\
\hline 5 & 11 & 0 & $\dagger$ & - & & $100 \mathrm{p} \cdot 100$ & $\begin{array}{l}\text { Mai-Juin } 1965 \\
\text { Dócembre } 1965\end{array}$ \\
\hline 10 & 2 & 0 & 1 & - & $1 \operatorname{sur} 1$ & $"$ & Mai 1965 \\
\hline 20 & 40 & 0 & 1 & - & 1 sur 1 & $"$ & Janvier 1965 \\
\hline 40 & 38 & 0 & 1 & - & 1 sur 1 & $"$ & Janvier 1965 \\
\hline \multicolumn{2}{|c|}{ Voie sous-cutanée. } & & & & & & $\therefore$ \\
\hline 5 & 38 & 0 & 84 & - & $6 \operatorname{sur} 6$ & $100 \mathrm{p} \cdot 100$ & $\begin{array}{l}\text { Novembre- } \\
\text { Décembre } 1965\end{array}$ \\
\hline 8 & 5 & 0 & - & - & 1 sux 1 & $"$ & $\begin{array}{ll}\text { Ners } & 1966 \\
\text { Juin } & 1965\end{array}$ \\
\hline 10 & - & - & 50 & $n-$ & 1 sux 1 & $n$ & Juin \\
\hline
\end{tabular}

* = dans une goutte de suspension aqueuse provenant des boîteg de Pétri ayent pervi. aux coprocultures.

TABLEAU NOTI

Action du létranisole sur divers filariidae du zébu. Nombra d'animaux déparasités après traitement.

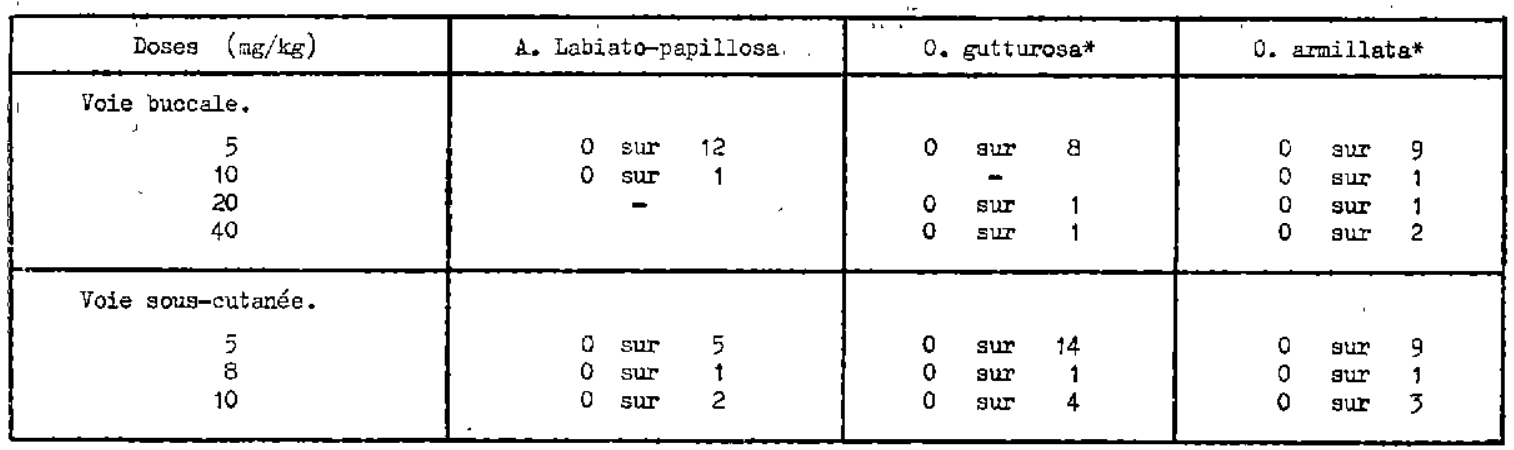

* Les Onchocerques sont soigneusement retirés des tissus où ils se trouvent et mis dens du sérum physiologique tiède, de façon à savoir s'ils sont encore en vie ou non. Un examen ultérieur au microscope, ap rès éclaircissement, donne des indications sur la dégénérescence éventuella des parasites.

TABLEAU HOXII

Action du Tétramieole sur les formes $\mathrm{L}_{4}$ tntranodulairea.

\begin{tabular}{|c|c|c|c|c|}
\hline $\begin{array}{r}\text { Doseg } \\
(\mathrm{mg} / \mathrm{kg})\end{array}$ & $\begin{array}{c}\text { Nombre d'animaux } \\
\text { parasités }\end{array}$ & $\begin{array}{l}\text { Nombre total de } \\
\text { nodules caecaux }\end{array}$ & $\begin{array}{l}\text { Nombre de } \mathrm{I}_{4} \text { encore } \\
\text { viventes a } I^{\prime} \text { autopsie }\end{array}$ & $\begin{array}{l}\text { Epoque des } \\
\text { traitements }\end{array}$ \\
\hline $\begin{array}{c}\text { Vole } \\
5 \\
10 \\
40\end{array}$ & $\begin{array}{l}2 \\
1 \\
3\end{array}$ & $\begin{array}{l}0 \\
1 \\
3\end{array}$ & $\begin{array}{l}3 \\
1 \\
1\end{array}$ & $\begin{array}{l}\text { Novembre-Décembre } 1965 \\
\text { Maż } 1965 \\
\text { Janvier } 1965\end{array}$ \\
\hline $\begin{array}{l}\text { Voie } \\
5\end{array}$ & Ltanée. & 5 & 2 & Décembre 1965 \\
\hline
\end{tabular}


b) D'une façon générale, sur des lots d'animaux faiblement ou moyennement infestés, le médicament entraîne, 10 jours après le traitement, la disparition de la quasi-totalifé des œufs de Nématodes intestinaux.

c) A $5 \mathrm{mg} / \mathrm{kg}$, que ce soit par la voie buccale ou par la voie sous-cutanée, le 16.535 R. P. assure l'élimination à 100 p. 100 de Bunostomum phlebotomum, Bosicola radiatum et Haemoncus 'contortus adultes, mûrs et immatures. L'effet sur Cooperia pectinata et Cooperia punctata est moins complet ( $90-95$ p. 100) environ.

d) Pour Bluckleyuris globulosa, les renseignements recueillis, trop fragmentaires, ne permettent pas de tirer des conclusions définitives. Comme chez le mouton, l'action serait plutôt irrégulière.

e) Strongylöides papillosus, contrairement à ce qui se passe chez le mouton (GRABER, 1966), serait déjà détruit vers $5 \mathrm{mg} / \mathrm{kg}$. Les essais n'ont porté que sur des infestations très réduites : ils devront donc être confirmés.

f) Les formes intranodulaires $L_{4}$ de Bosicola radiatum ne sont que peu touchées par l'anthelminthique et la plupart d'entre elies demeurent vivantes dans les nodules cæcaux.

9) Quant aux formes larvaires immatures de Cooperia pectinata et de Cooperio punctata, un certain nombre d'entre elles persistent à $5 \mathrm{mg} / \mathrm{kg}$ par la voie buccale.

A titre de comparaison, le Tableau No XIII donne, chez les jeunes zébus du Tchad ef de R. C. A., les pourcentages de réduction obtenus après traitement, au Thiabendazole (GRABER, 1965) et au Tétramisole :

Le Tétramisole semble supérieur au Thiabendazole lorsque l'on est en présence d'infestation par Bosicola radiatum et Bunostomum phlebotomum adultes mûrs ou immatures. Il est un peu moins actif sur Cooperia pectinato et sur Cooperia puncfoto. La valeur des deux médicaments est à peu de chose près équivalente quand il s'agit d'Hoemoncus contortus ou de formes larvaires $L_{4}$ intranodulaires de Bosicola radiafum.

Dans la lutte contre les Nématodes sévissant en milieu tropical, la dose de $5 \mathrm{mg} / \mathrm{kg}$, quelle que soit la voie d'introduction, paraît devorr être recommandée, car elle est capable à 90-95 p. 100 de chasser les principaux Nématodes entrant dans la composition des associations parasitaires, si nombreuses au Tchad ef en R. C. A. Par contre, si l'on désire absolument supprimer tous les parasites, il fout alors utiliser la dose de $8-10 \mathrm{mg} / \mathrm{kg}$.

\section{ACTIVITÉS DU MÉDICAMENT}

Comme chez le mouton (GRABER, 1966), l'évacuation des parasites débute aussitôt après la fin du traitement. Dans 93 p. 100 des cas, elle est terminée au bout de 24 heures.

Au-delà de 48 heures, les Bosicola, Bunostomum et Haemoncus adultes ont été tous rejetés à l'extérieur et les possibilités d'infestation d'un pâturage neuf par les larves $L_{3}$ de ces Nématodes sont pratiquement nulles.

\section{CONSÉQUENCES. DU TRAITEMENT SUR LA SANTÉ DE L'ANIMALL}

\section{A. - CONSÉQUENCE VISIBLE}

A la dose de $5 \mathrm{mg} / \mathrm{kg}$, le médicament est très bien supporté et ne cause pas de perturbations sensibles sur le comportement de l'animal. L'appétit croìt rapidement et le foin tend à être consommé en plus grande quantité par les animaux traités que par les témoins.

\section{B. - NUMÉRATIONS GLOBULAIRES FORMULES LEUCOCYTAIRES}

Le traitement au Tétramisole ne détermine pas de modifications appréciables du nombre d'hématies qui, au bout de 5 jours, a tendance, soit à demeurer stable, soit à augmenter progressivement ( $2 / 3$ des cas).

Les leucocytes varient dans d'étroites limites.

\section{C. - PROTÉINES DU SANG}

Les recherches (*) ont porté sur cinq animaux ayant reçu $5 \mathrm{mg} / \mathrm{kg}$ par la bouche en décemNET.

*) En collaboration avec M. QUEVAL ef Mme BRU. 


\section{TABLEAU NOXIII}

Comparaison du pouvoir anthelmanthique du Thiabendazole et du Tétrarisole (Nématodes adultes mûrg et immatures)

\begin{tabular}{|c|c|c|c|}
\hline Espèces en cause & $\begin{array}{l}\text { Thiabendazole } \\
70-80 \mathrm{mg} / \mathrm{kg}\end{array}$ & & $\begin{array}{l}\text { Tétramigole } \\
(5 \mathrm{mg} / \mathrm{kg})\end{array}$ \\
\hline $\begin{array}{l}\text { Bosicola radiatum } \\
\text { Bunostomum phlebotomum }\end{array}$ & $\begin{array}{l}84 \text { aे } 97,3 \mathrm{p} \cdot 11 \\
\dot{44} \text { aे } 88\end{array}$ & $\begin{array}{c}100 \\
11\end{array}$ & $\begin{array}{l}100 p \cdot 100 \\
100\end{array}$ \\
\hline $\begin{array}{l}\text { Cooperia peotinata } \\
\text { Cooperia punctata }\end{array}$ & 90 à 98,2 & $n$ & 90 à $95 "$ \\
\hline Haemoncus contortus & $93,4 a ̀ \quad 100$ & $"$ & 100 \\
\hline Buckleyuris globulose & 10 à 30 & " & 10 à $50 "(?)$ \\
\hline
\end{tabular}

GRAPHIQUE $n^{\circ} I$ : Comparaison entre le pourcentoge défficacite du Tétramisole et du Thiabendazole sur divers Nématodes gastro-intestınaux du zébu



Te $=$ Tétromisole $5 \mathrm{mg} / \mathbf{k g r}$

Th $=$ Thiabendazole $70-80 \mathrm{mg} / \mathrm{kgr}$ sur le terrain 
GRAPHIQUE $n^{\circ}$ II

RELEVES CLIMATIOUES

températures en $0: \mathrm{C}$

$-----\infty$ degré hygrométrique

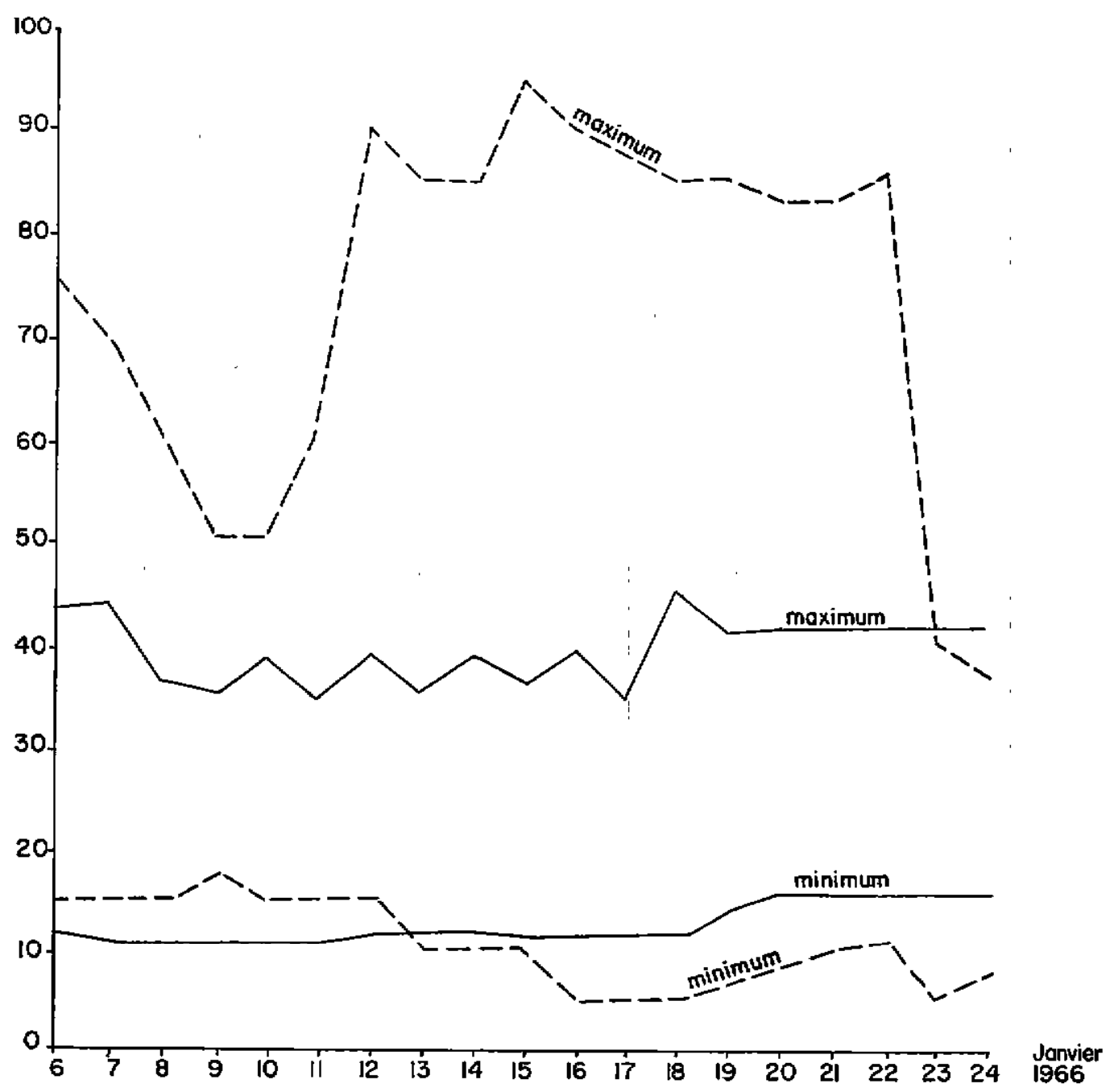


bre 1965 (Tableau No XIV). Les variations subies par les Protéines du sang, dans ce lot composé d'animaux très parasités et très anémiés, sont de faible ampleur ef peu significatives. Les Protéines totales diminuent de moins de 1 p. 100 au profit des albumines et le rapport Albumine/. Globuline passe alors de 0,40 à 0,42.

\section{VALEUR ÉCONOMIQUE DU MÉDICAMENT}

Elle se mesure aux gains de poids évalués à la bascule.

10 En étable sur une période de six jours.
L'essai a porté sur 22 animaux, jeunes et âgés, traités d̀ $5 \mathrm{mg} / \mathrm{kg}$. Ils ont été nourris de foin à base de Brochiaria, d'Echinochloa et de Panicum et entretenus dans des stalles cimentées.

Le gain de poids est comparable dans les deux'cas.

\section{2o Sur le terrain.}

Cinq jeunes taurillons diversement parasités par Cooperia, Bosicola, Bunostomum et Haemoncus ont été remis, après traitement au Tétramisole par la voie buccale $(5 \mathrm{mg} / \mathrm{kg})$, sur un pâturage de saison sèche maigre, clairsemé et de mauvaise qualité (herbes grossières sans chaumes de mil). Cing autres bouvilions ont servi de témoins.

TABLEAU NOXV

Tétramisole $5 \mathrm{mg} / \mathrm{kg}$ - Protéines du sang.

\begin{tabular}{|c|c|c|c|c|c|c|c|c|}
\hline Bouvillons $n^{0}$ & 1 & 2 & 1747 & 976 & 2981 & 2997 & \multicolumn{2}{|l|}{ Moyenne } \\
\hline $\begin{array}{l}\text { Protéines totalea } \\
\text { Avant traitement } \\
\text { Après traltement* }\end{array}$ & $\begin{array}{l}73,50 \\
73,50\end{array}$ & $\begin{array}{l}73,50 \\
69,82\end{array}$ & $\begin{array}{l}79,38 \\
73,50\end{array}$ & $\begin{array}{l}76,44 \\
79,38\end{array}$ & $\begin{array}{l}73,50 \\
76,44\end{array}$ & $\begin{array}{l}69,82 \\
69,82 \\
\end{array}$ & $\begin{array}{l}74,34 \\
73,74-0,9 \mathrm{p} .\end{array}$ & \\
\hline $\begin{array}{l}\text { Globullnes } \\
\text { Avant traitement } \\
\text { Aprèa traitement }\end{array}$ & $\begin{array}{l}21,31 \\
22,20\end{array}$ & $\begin{array}{l}22,20 \\
20,66\end{array}$ & $\begin{array}{l}22,20 \\
21,43\end{array}$ & $\begin{array}{l}20,67 \\
22,20\end{array}$ & $\begin{array}{l}21,31 \\
22,20\end{array}$ & $\begin{array}{l}21,43 \\
22,96\end{array}$ & $\begin{array}{l}21,52 \\
21,94+1,90\end{array}$ & $"$ \\
\hline $\begin{array}{l}\text { Albumines } \\
\text { Avant traitement } \\
\text { Aprés traitement }\end{array}$ & $\begin{array}{l}52,19 \\
51,30\end{array}$ & $\begin{array}{l}51,30 \\
49,16\end{array}$ & $\begin{array}{l}57,18 \\
52,07\end{array}$ & $\begin{array}{l}55,77 \\
57,18\end{array}$ & $\begin{array}{l}52,19 \\
54,24\end{array}$ & $\begin{array}{l}48,39 \\
46,86\end{array}$ & $\begin{array}{l}52,83 \\
51,80-2\end{array}$ & $"$ \\
\hline
\end{tabular}

* a jours après l'administration du Tétramisole.

TABLEAU NOXV

Augmentation de poida en étable

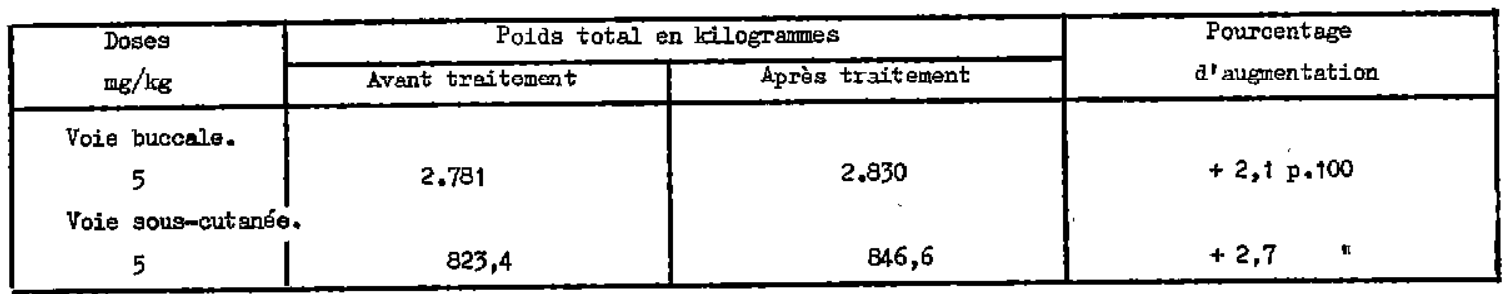

TABLEAU HO $N \mathrm{NT}$

Augmentation de poids sur le terrain

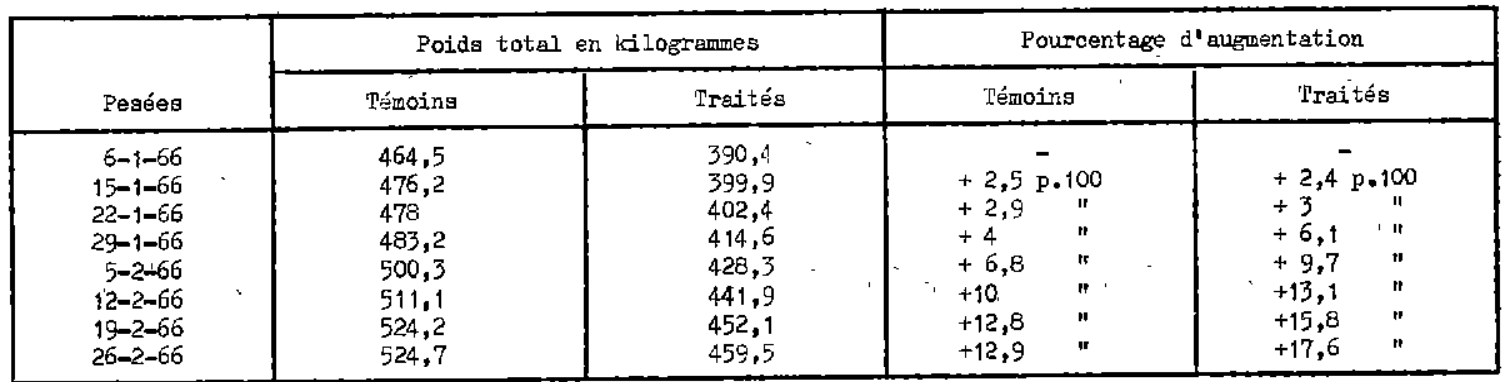


GRAPHIQUE $n$ \%III

TETRAMISOLE-Gains de Poids

témoins|

-

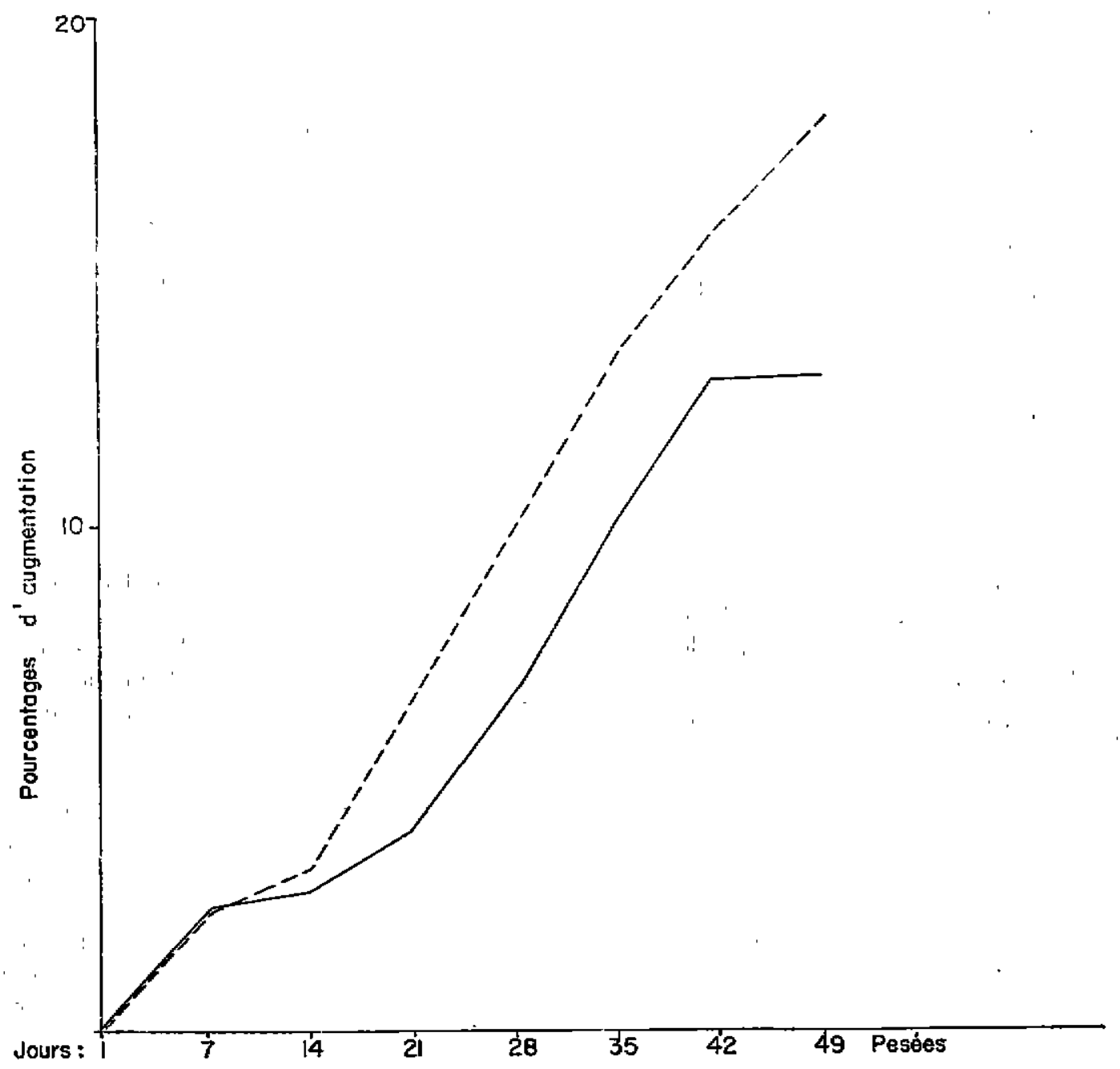


Ils ont été pesés régulièrement une fois par semaine du 6-1-1966 au 26-2-66 (Tablequ No XVI). L'expérience a été arrêtée fin février, les parcours valables étant alors trop éloignés du Laboratoire.

Les résultats sont assez bons. Chez les animaux traltés, le gain de poids par rapport aux témoins est de $+4,5$ p. 100 en sept semaines. L'état des animauxs'améliore considérablement. L'augmentation de poids est sensible chez tous les animaux, alors que, chez les témoins, elle n'est le fait que de deux bouvillons, le poids des trois autres demeurant stable.

Le 16.535 R. P. présente donc, en matière d'élevage, un intérêt économique indéniable.

\section{TOXICITÉ}

Des doses progressivement croissantes ont été expérimentées :

Les doses progressivement crolssarites ont été expérımentées.

\begin{tabular}{|c|c|c|c|}
\hline $\begin{array}{l}\text { Doses } \\
\mathrm{mg} / \mathrm{kg}\end{array}$ & $\begin{array}{c}\text { Nombre d'animaux } \\
\text { utilisés }\end{array}$ & Mortalité & Epoque des essais thérapeutiques \\
\hline $\begin{array}{l}\text { Woie bucaale. } \\
\qquad \begin{array}{c}5 \\
10 \\
20 \\
40 \\
70 \\
75 \\
100\end{array}\end{array}$ & $\begin{array}{l}23 \\
5 \\
3 \\
3 \\
3 \\
2 \\
2\end{array}$ & $\begin{array}{c}0 \\
0 \\
0 \\
0 \\
0 \\
1 \text { sur } 3 \\
2 \text { sur } 2 \\
2 \text { sur } 2\end{array}$ & $\begin{array}{l}\text { Mai-Novedbre-Décembre } 1965 \\
\text { Mars-Avril } 1966 \\
\text { Mai } 1965 \\
\text { Janvier } 1965 \\
\text { Junvier } 1965 \\
\text { Mal } 1965 \\
\text { Avril } 1966 \\
\text { Mat } 1965\end{array}$ \\
\hline $\begin{array}{c}\text { Voie sous-Cut } \\
5 \\
8 \\
10 \\
40 \\
50 \\
60 \\
65 \\
70 \\
80\end{array}$ & $\begin{array}{r}17 \\
1 \\
3 \\
2 \\
2 \\
2 \\
2 \\
2 \\
3\end{array}$ & $\begin{array}{l}0 \\
0 \\
0 \\
0 \\
2 \operatorname{sur} 2 \\
2 \operatorname{sur} 2 \\
2 \operatorname{sur} 2 \\
2 \operatorname{sur} 2 \\
3 \operatorname{sur} 3\end{array}$ & $\begin{array}{l}\text { Nai-Juin-Novembre-Décembre } 1965 \\
\text { Juin } 1965 \\
\text { Juin } 1965 \\
\text { Avril } 1966 \\
\text { Avril } 1966 \\
\text { Avril } 1966 \\
\text { Avil } 1966 \\
\text { Juin } 1965 \\
\text { Juin } 1965\end{array}$ \\
\hline
\end{tabular}

- Par la voie buccale, le Tétramisole tue tous les tourilions vers $75 \mathrm{mg} / \mathrm{kg}$. Le coefficient chimiothérapıque, si l'on s'en tient à la dose de $5 \mathrm{mg} / \mathrm{kg}$, est alors de 15 .

- Par la voie sous-cutanée, le 16.535 R. P. est beaucoup plus toxique, puisque les premiers accidents martels apparaissent entre 40 et $50 \mathrm{mg} / \mathrm{kg}$. Le coefficient chimiathérapique oscille autour de 8-10.

La marge de sécurité, chez le zébu tchadien, est donc importante lorsque le médicament est administré par la voie buccale. Par la voie souscutanée, les résultats sont moins favorables.

Dans cette espèce, la toxicité du Tétramısole ne semble pas supérieure à celle du Thiabendazole : avec cet anthelminthique, des accidents mortels (GRABER, 1965) se font déjà jour vers $750 \mathrm{mg} / \mathrm{kg}$ chez le bouvillon ( $C / T=7,5$ ) et chez le veau de lait (GRABER en préparation) vers $640 \mathrm{mg} / \mathrm{kg}(C / T=8)$. L'intoxication au Tétramisole évolue de la même façon que chez le mouton (GRABER, 1966) et les signes sont comparables. Tout au plus doit-on noter une plus grande propension à l'excitabilité. Les animaux qui résıstent ( $70 \mathrm{mg} / \mathrm{kg}$, voie buccale) deviennent méchants, chargent constamment sans cause apparente et extériorisent des perversions de goût curieuses. A $40 \mathrm{mg} / \mathrm{kg}$ (voie sous-cutanée) les mêmes manifestations apparaissent, mais elles sont fugaces. Il est bon de remarquer que, parmi les vaches traitées, plusieurs d'entre elles portaient des embryons de quatre mois. Aucun avortement n'a été constaté. 


\section{CONCLUSIONS}

Lors d'essais effectués en 1965-1966 sur 107 zébus, jeunes et adultes, originaires des régions Ouest du Tchad, il a été constaté.

$1^{\circ}$ Que le Tétramisole, quelle que soit la dose utilisée, est complètement inactif sur Fosciola gigantica des canaux biliaires, Poromphistomum microbothrum ef Carmyerius papillatus de la panse, Schistosoma bovis des veines hépatiques et mésentériques, les grands Cestodes de l'intestin (Moniezia benedeni et Thysaniezio ovilla) et Cysticercus bovis.

$2^{0} \mathrm{ll}$ en est de même pour les. Filaires du péritoine (Artıonema labiato-popillosa), de l'aorte (Onchocerca armillota) et du ligament cervical (Onchocerca gutturosa).

$3^{\circ}$ Sur les associations à base de Bosicola radiatum, Bonosiomum phlebotomum, Haemoncus contortus, Cooperia punctata et Cooperia pectinafa adultes mûrs ou immatures, la dose de $5 \mathrm{mg} / \mathrm{kg}$ administrée par la voie sous-cutanée ou par la voie buccale sans préparation spéciale (diète) assure l'expulsion d'environ 90-95 p. 100 des Nématodes gastro-intestinaux entrant dans la composition des associations parasitaires.

40 A la même dose, les larves $L_{4}$ de Bosicola rodiatum profondément enkystées dans la muqueuse intestinale ne paraissent pas touchées par le Tétramisole. Quelques formes larvaires immatures de Cooperio pectinata et de Cooperia punctata persistent également après traitement.

$5^{\circ}$ Le médicament, pour le zébu tchadien, semble assez peu toxique. Le coefficient chimiothérapique va de 8-10 (voie sous-cutanée) ò 15 (voie buccale). Cependant, vers $40 \mathrm{mg} / \mathrm{kg}$, certains signes d'intoxication (excitabilité) se manifestent. Fugaces, ils ne durent pas.

A $5 \mathrm{mg} / \mathrm{kg}$, les numérations globulaires et le dosage des protéines du sang ne montrent pas de changements appréciables.

60 En milieu tropical sec, le Tétramisole en chassant les Nématodes les plus fréquents, entraîne une reprise de poids rapide qui peut être chiffrée - dans les plus mauvaises conditions imaginables, comme c'est le cas pour la saison 1965-1966 - à + 17,6 p. 100 (témoins 12, 9 p. 100). L'état général des animaux s'améliore considérablement.

Le Tétramisole - comme le Thiabendazole -constifue done un anthelminthique d'une grande valeur économique.

\section{REMERCIEMENTS}

Nous tenons à remercier vivement la société Rhône-Poulenc pour sa contribution appréciable à la réalisation de ces essais, en particulier MM. QUENTIN et SIRY de la Direction scientifique.

\section{SUMMARY}

Study of the anthelmintic power of Tetramisole (16.535 R. P.)

on various helminths of zebu cattle in Chad Republic

Trials have been made to test the therapeutic property of Tetramisole in 106 young and adult zebus from Western Region of Chad. Tetramisale, at the dose of $5 \mathrm{mg} / \mathrm{kg}$ given without diet, per os or by subcutaneous injection was active in 90-95 p. 100 of the cases against mature or immature sloges of Bosicolo radiatum, Bunostomum phlebotomum. Haemoncus contortus, Cooperia pectinata et Cooperio punctata. The $\mathrm{L}_{4}$ stages of Bosicola radiatum, which are encysted in the intestinal mucous membrane, were resistant to this treatment. Some larval stages of Cooperio were also resistant.

This drug is relatively safe for the zebu, since its chemotherapeutic coefficient ranges between 8 and 10 (subcutaneous route) to 15 (per os).

At the therapeutic dose, which is well tolerated, the improvement of the condition is quick and, after seven weeks, under very poor nutritional conditions, the weight increased by 17,6 p. 100 (12,9 p. 100 in reference animals).

The economical interest of 16.535 R. P. is obvious. 


\section{RESUMEN}

Estudio del poder antihelmintico del Tetramisole (16.535 R. P.) en varios helmíntos del cebú de la República del Chad

Se efecfuaron ensayos para demostrar la actividad terapéutica del Tetramisole en 106 cebues, jovenes y adultos, de las regiones del Oeste de la República del Chad, Una dosis de $5 \mathrm{mg} / \mathrm{kg}$ de este medicamento administrada sin dieta, per os o por inyección subcutánea, seria suficiente para destruir un termino medio de $90-95$ p. 100 de los grupos adultos maduros o inmaduros de Bosicola radiatum, Bunostamum phlebotomum, Haemoncus contortus, Cooperio pectinata y Cooperia punctota,

Las formas $L_{4}$ de Bosicolo radiafum enquistadas en la mucosa intestinal resisten al tratamienta. Algunas formas larvarias de Cooperia persisten igualmente.

El medicamento es relativamente poco toxica para el cebú, ya que el coeficiente quimioterapéutico vario de 8-10 (inyección subcutánea) a 15 (per os).

Se mejora el estado general muy rapidamente en la dosis terapéutica, bien tolerada ; siefe semanas más tarde, con muy malas condiciones alimenticias la ganancia de peso llega a $+17,6$ (testigos : $+12,9$ ). El 16.535 R. P. es de un interés éconómico cierto.

\section{BIBLIOGRAPHIE}

1. GRABER (M.). - Parasitoses internes. Rap. onn. Ministère agriculture ef production onimale Tchod, Fasc. VII, 1964, 89-127.

2. GRABER (M.). - Etude dans certaines conditions africaines de l'action antiparasitaire du Thiabendazole sur divers Helminthes des animaux domestiques. I. Helminthes du zébu. Rev. Elev. Med. Vét. Pays Trop., 1965, 18, I, 39-58.

3. GRABER (M.). - Action d'un nouvel anthelminthique, le Tétramisole (16.575 R. P.) sur divers Helminthes du mouton de la République du Tchad. Rev. Elev. Med. Vét. Pays Trop., 1966, 19 (sous presse):
4. THIENPONT (D.)', VANPARIJS (O. F. J.), RAEMAEKERS (A. H. M.), VANDENBERK (J.), DEMOEN (J. A.), ALLEWIJN (F. T. N.) MARSBOOM (R. P. H.), NIEMEGEERS (C. J. E.), SCHELLEKENS (K. H. L.) and JANSSEN (A. J.).'- Tétramisole ( $R$, 8299), a new, potent broad spectrum anthelmintic. Nature, 1966, 209, 1084-86.

5. WALLEY (J. K.). - Tétramisole (dl 2, 3, 5, 6tetrahydro-6-phenyl-imidazo (2, 1-b) thiazole hydrochloride. Nilwern) in the treatment of gastro-intestinal worms and lungworms in domestic animals. I. Sheep and goats. Vet. Rec., 1966, 78, 12, 406-414. 